\title{
Time-course of upper respiratory tract viral infection and COPD exacerbation
}

\author{
Daiana Stolz ${ }^{1,2}$, Eleni Papakonstantinou ${ }^{1,2}$, Leticia Grize ${ }^{3,4}$, Daniel Schilter ${ }^{5}$, \\ Werner Strobel ${ }^{1,2}$, Renaud Louis $^{6}$, Christian Schindler $^{3,4}$, Hans H. Hirsch $^{2,7}$ and \\ Michael Tamm ${ }^{1,2,7}$
}

Affiliations: ${ }^{1}$ Clinic of Respiratory Medicine and Pulmonary Cell Research, University Hospital Basel, University of Basel, Basel, Switzerland. ${ }^{2}$ Dept of Biomedicine, University of Basel, Basel, Switzerland. ${ }^{3}$ University of Basel, Basel, Switzerland. ${ }^{4}$ Swiss Tropical and Public Health Institute, Basel, Switzerland. ${ }^{5}$ Lindenhof Hospital, Bern, Switzerland. ${ }^{6}$ Pneumology Dept, University of Liege, CHU Liege, Liege, Belgium. ${ }^{7}$ Both authors contributed equally.

Correspondence: Daiana Stolz, Clinic of Pulmonary Medicine and Respiratory Cell Research, University Hospital Basel, Petersgraben 4, CH-4031 Basel, Switzerland. E-mail: daiana.stolzQusb.ch

@ERSpublications

The presence of viruses in patients with stable COPD is rare. URTI viruses were not per se associated with an increased risk of exacerbation. URTI is associated with worsening of quality of life and lung function independently of exacerbation. http://bit.ly/30jGm5N

Cite this article as: Stolz D, Papakonstantinou E, Grize L, et al. Time-course of upper respiratory tract viral infection and COPD exacerbation. Eur Respir J 2019; 54: 1900407 [https://doi.org/10.1183/ 13993003.00407-2019].

ABSTRACT Viral respiratory tract infections have been implicated as the predominant risk factor for acute exacerbations of chronic obstructive pulmonary disease (AECOPD). We aimed to evaluate, longitudinally, the association between upper respiratory tract infections (URTI) caused by viruses and AECOPD.

Detection of 18 viruses was performed in naso- and oropharyngeal swabs from 450 COPD patients (Global Initiative for Chronic Obstructive Lung Disease stages 2-4) who were followed for a mean of 27 months. Swabs were taken during stable periods $(n=1909)$, at URTI onset $(n=391), 10$ days after the URTI ( $\mathrm{n}=356)$ and during an AECOPD $(\mathrm{n}=177)$ and tested using a multiplex nucleic acid amplification test.

Evidence of at least one respiratory virus was significantly higher at URTI onset (52.7\%), 10 days after the URTI $(15.2 \%)$ and during an AECOPD $(38.4 \%)$, compared with the stable period $(5.3 \%, \mathrm{p}<0.001)$. During stable visits, rhinovirus accounted for $54.2 \%$ of all viral infections, followed by coronavirus (20.5\%). None of the viruses were identified in two consecutive stable visits. Patients with a viral infection at URTI onset did not have a higher incidence of exacerbation than patients without viral infection $(\mathrm{p}=0.993)$. The incidence of any viral infection during an AECOPD was similar between URTI-related AECOPD and non-URTI-related AECOPD ( $p=0.359$ ). Only $24 \%$ of the patients that had a URTI-related AECOPD had the same virus at URTI onset and during an AECOPD. Detection of parainfluenza 3 at URTI onset was associated with a higher risk of an AECOPD ( $p=0.003)$. Rhinovirus and coronavirus were the most frequently detected viruses during AECOPD visits, accounting for $35.7 \%$ and $25.9 \%$ of all viral infections, respectively.

The prevalence of viral infection during the stable period of COPD was low. The risk of exacerbation following the onset of URTI symptoms depends on the particular virus associated with the event and was significant only for parainfluenza 3 .

This article has supplementary material available from erj.ersjournals.com

Received: 27 Feb 2019 | Accepted after revision: 28 June 2019

Copyright OERS 2019 


\section{Introduction}

Chronic obstructive pulmonary disease (COPD) is the third leading cause of death worldwide, with its prevalence and mortality expected to increase in the next decades [1]. Exacerbations are significant events in COPD that affect the progression of the disease and contribute to morbidity, mortality, hospital admission and healthcare costs [2]. It is widely recognised that bacterial or viral infections can trigger acute exacerbations of COPD (AECOPD) given that $40 \%-80 \%$ of AECOPD that frequently require hospitalisation are attributed to viral respiratory tract infections [3]. AECOPD associated with symptoms of a common cold have been shown to have more sudden onset and longer recovery times than AECOPD without cold symptoms [4]. COPD patients who have more frequent exacerbations experience nearly double the number of colds compared to patients experiencing fewer exacerbations [5]. It has also been shown that the presence of cold symptoms is associated with an increased risk of AECOPD [6].

Despite the recent development of more specific and sensitive diagnostic methods that use PCR for the detection of respiratory viruses, the presence of a virus is usually identified in less than half of upper respiratory tract infections (URTIs), questioning the incidence of viral infections in URTIs and to what extent they contribute to AECOPD. There are scarce longitudinal data evaluating COPD both during stable and exacerbation periods [7,8], but these reports appoint human rhinoviruses as the most common aetiology for viral exacerbations [9]. Several other viruses may also cause disease in patients with COPD, including coronavirus, influenza, parainfluenza 1-3, respiratory syncytial virus (RSV), adenovirus and human metapneumovirus [10-12]. However, because URTI symptoms often precede COPD exacerbations it is difficult to precisely define the proportion of exacerbations caused by viruses that were originally associated with the URTI. Therefore, clinical studies restricted to sampling for viruses during COPD exacerbations may fail to detect viruses despite highly sensitive PCR technology [10].

In the present study, a well-characterised cohort of 450 patients was evaluated longitudinally for the presence of viral infections by multiplex PCR during stable state, at the onset of URTI, 10 days after the URTI and during an AECOPD. The aim of the study was to evaluate, longitudinally, the association between URTIs caused by viruses and AECOPD. We hypothesise that patients with URTIs have a higher incidence of AECOPD.

\section{Methods}

Study design

In the present study we analysed the co-primary aim of the PREVENT (Preventing Viral Exacerbation of Chronic Obstructive Pulmonary Disease in Upper Respiratory Tract Infection) study, an investigatorinitiated and -driven multicentre, controlled trial (ISRCTN45572998) [13] that evaluated, longitudinally, the association between URTI caused by viruses and AECOPD. The primary co-aim of the PREVENT study was to evaluate whether, as compared to placebo, intensified combination therapy with inhaled corticosteroids (ICS) and long-acting $\beta 2$-agonists (LABA) at the onset of URTI symptoms decreased the incidence of AECOPD in patients receiving a low maintenance dose of ICS/LABA [13]. The study was approved by each of the institutional review boards (EKBB 306/10) and was conducted in accordance with the ethical principles stated in the Declaration of Helsinki and the guidelines on good clinical practice. All patients provided written informed consent for their participation in the study.

\section{Patients}

450 COPD patients aged $>40$ years and with a smoking history of $\geqslant 10$ pack-years were enrolled. Inclusion criteria were a post-bronchodilator forced expiratory volume in $1 \mathrm{~s}(\mathrm{FEV} 1)<80 \%$ of the predicted value, a post-bronchodilator ratio of FEV1 to forced vital capacity (FVC) $<0.7$ (Global Initiative for Chronic Obstructive Lung Disease (GOLD) stages 2-4) and clinically relevant disease, as defined by a history of at least one exacerbation in the previous 12 months. Exclusion criteria were the presence of a pulmonary condition other than COPD as the main respiratory disease, e.g. bronchiectasis or asthma; a rapid lethal disease, e.g. bronchial carcinoma, advanced heart failure or end-stage renal failure; severe immunosuppression, including manifested AIDS, organ transplantation or neutropenia $\left(<500 \times 10^{9}\right.$ cells. $\left.\mathrm{L}^{-1}\right)$; pregnancy or breast feeding; and a known allergy or intolerance to the study medication.

\section{Procedures}

At inclusion, all patients were assigned to a low maintenance dose of ICS/LABA (budesonide $200 \mu \mathrm{g} /$ formoterol $6 \mu \mathrm{g}$, twice daily). This treatment was continued for the duration of the study, while other concomitant medication, including long-acting muscarinic antagonist, was left unchanged. In addition, each patient was block-randomised 1:1 to receive either an intensified dosage of the combination ICS/ LABA (budesonide $400 \mu \mathrm{g} /$ formoterol $12 \mu \mathrm{g}$ ) or placebo. Patients were instructed to start using the intensified dosage of ICS/LABA at the onset of the URTI, twice daily, for 10 days. 
Clinical history, vital signs and oxygen saturation were evaluated at baseline. Patients were asked to answer the following symptoms and quality of life questionnaires: the COPD Assessment Test (CAT), the modified Medical Research Council (mMRC) dyspnoea scale, the Wisconsin Upper Respiratory Symptom Survey-21 (WURSS-21) and the St George's Respiratory Questionnaire (SGRQ). Serum and EDTA-plasma samples, spontaneous or induced sputum and nasopharyngeal swabs were collected. Body plethysmography, 6-min walk test and FeNO measurements were administered by trained technicians using properly calibrated instruments according to European Respiratory Society (ERS)/American Thoracic Society (ATS) guidelines. Nasopharyngeal and oral swabs were also collected at the study centre at stable visits.

When patients had a URTI that was defined as new or increased coryzal symptoms (one or more of runny or blocked nose, post-nasal drip and sneezing) for at least $12 \mathrm{~h}$, they were instructed to collect nasopharyngeal and oral swabs using a technique that was demonstrated to the patients. Swabs were sent to the study centre by mail. Patients were also asked to contact the study centre within 10 days of URTI onset or in case of an exacerbation.

Patients attended a clinical visit 10 days after the onset of an URTI. At this URTI-follow-up visit, vital signs, oxygen saturation, symptoms scores, medication and healthcare resource use were assessed. Spontaneous or induced sputum, nasopharyngeal swabs and serum/EDTA-plasma samples were collected. Lung function tests and FeNO measurements were performed according to ERS/ATS guidelines.

In case of an AECOPD, the patient could seek medical care from a general practitioner (GP) or at the study centre. Symptoms recorded were termed major (dyspnoea, sputum volume or sputum purulence) and minor (cough, wheeze, sore throat or coryza). Exacerbations were defined as mild (requiring medical care and increased dose of short-acting $\beta 2$-agonist), moderate (requiring either antibiotics and/or parenteral corticosteroids) and severe (requiring hospitalisation). In all cases, patients were asked to immediately contact the study centre and attend a clinical visit. At this visit, vital signs, oxygen saturation, symptoms scores, medication and healthcare resource use were assessed. Spontaneous or induced sputum, nasopharyngeal swabs and serum/EDTA-plasma samples were collected. Lung function tests and FeNO measurements were performed. In all but mild exacerbations, chest radiography for pneumonia exclusion was strongly suggested. Data for patients seeking care outside the study centre were obtained by reviewing the medical records from the GP or other healthcare institutions. Management of exacerbations of COPD, e.g. antibiotic and steroid prescriptions, was left up to the discretion of the treating physician and was not influenced by study personnel. All patients who had an AECOPD had a follow-up visit 21 days after the AECOPD. At this follow-up visit, all patients were asked to fill in the CAT, the MMRC and the WURSS-21 questionnaires.

Every 6 weeks the health status of patients was verified by consulting the healthcare records of all involved providers and the patient's diaries (recording any URTI or exacerbation and use of steroids and antibiotics). Regular, scheduled interviews were performed every 6 weeks over the phone and every 6 months onsite throughout the study period.

\section{Viral and bacterial detection}

Detection of 18 viruses (adenovirus, influenza A/B H1-H3, parainfluenza 1-4, RSV A/B, rhinovirus/ enterovirus, coronavirus NL63/OC43/229E/HKU1, bocavirus and metapneumovirus) was performed in oral and nasopharyngeal swabs that were collected during stable periods $(n=1909)$, at URTI onset $(n=391)$, at URTI follow-up (10 days after the onset of URTI) $(n=356)$ and during AECOPD ( $\mathrm{n}=177)$ using a commercial multiplex nucleic acid amplification test (respiratory pathogen panel NxTAG-RPP; Luminex, MV's-Hertogenbosch, The Netherlands) [14], adding up to more than 50000 individual viral PCRs.

The analysis was performed at the Department of Virology, University Hospital Basel, by well-trained and certified personnel. A microorganism was defined as pathogenic if detected by the commercial multiplex PCR or by the culture-based method at the minimum concentration of $10^{3} \mathrm{CFU} \cdot \mathrm{mL}^{-1}$ or above $10^{4} \mathrm{CFU} \cdot \mathrm{mL}^{-1}$ (excluding mouth flora) depending on the bacterial species. Bacterial growth in sputum was analysed semi-quantitatively, as described by PODBIELSKi et al. [15].

\section{Statistical analysis}

The study data bank contained information on patient clinical characterisation and clinical tests and on the instruments used to determine their health status. All involved study centres entered the respective information for their patients in electronic case report forms and transferred them by password-secured website to the coordinating centre. The following software was used for data analyses: Statistical Analysis System 9.4 (SAS Institute, Cary, NC, USA), R for Windows (R Foundation for Statistical Computing, www.r-project.org) and STATA 14 (Stata Corp, College Station, TX, USA). 
For the descriptive statistics, categorical variables were summarised as counts and proportions (percentages) and continuous variables as mean $\pm \mathrm{SD}$, medians and ranges (minimum to maximum and interquartile range). The characteristics for different visits (stable, URTI, URTI follow-up and AECOPD) with and without viral infections were compared using a Chi-squared test or a Fisher's exact test as applicable if the characteristic was of a categorical nature. For continuous factors, the Mann-Whitney U-test was used to compare between viral infection and no infection.

The association of a viral infection (yes or no) or the presence of a specific virus with a respiratory symptom at different visit types was determined using mixed logistic regressions (because patients had several visits of the same type). The outcome for the models used in these regressions was a viral infection or the presence of a specific virus and the predictor was the respiratory symptom test score. The model was adjusted for age and sex as fixed effects and the effect of the patient was added as a random effect.

\section{Results}

\section{Patients}

From a total of 589 patients with COPD who were screened, 450 patients were eligible to be included in the study and 445 patients were sampled for PCR and included in the analysis (figure 1). During the study, 49 patients died, eight patients were relocated, 52 patients withdrew consent and 12 patients were lost to follow-up. From the remaining patients, 198 patients reported a total of 403 URTIs. A total of 187 AECOPD were observed in 118 patients. From these exacerbations, 42 were severe and required hospitalisation and four required a stay in the intensive care unit. Patients' mean follow-up was $714 \pm 339$ days, totalling 870 person-years.

Characteristics of the 445 patients that were included in the analysis are shown in table 1 . Patients were mostly male, were aged $>40$ years, had a smoking history of $\geqslant 10$ pack-years, were GOLD stage $2-4$ and had clinically relevant disease, as defined by a history of at least one exacerbation in the previous 12 months [13].

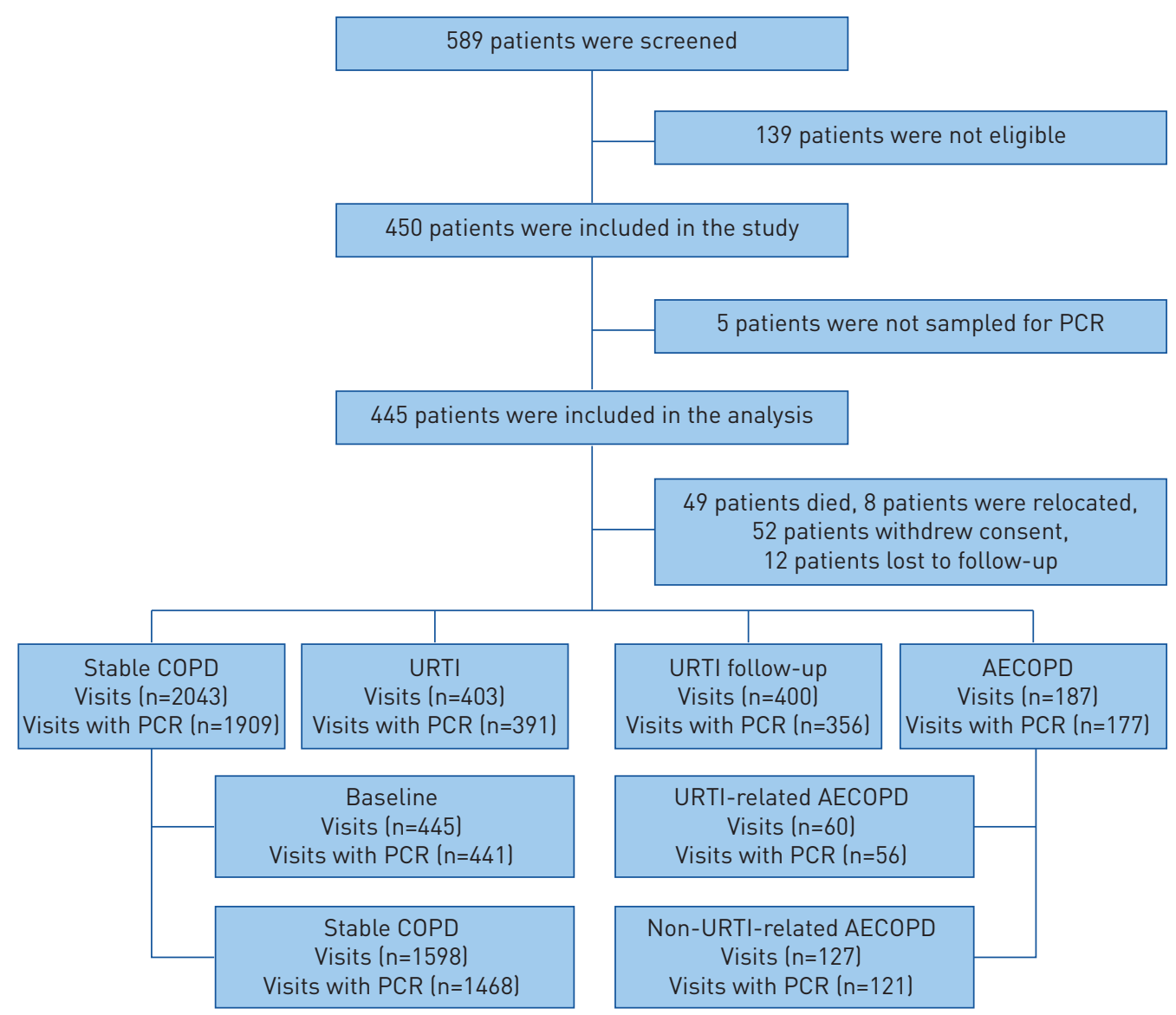

FIGURE 1 Consolidated Standards of Reporting Trials flow diagram. COPD: chronic obstructive pulmonary disease; URTI: upper respiratory tract infection; AECOPD: acute exacerbation of COPD; URTI-related AECOPD: AECOPD that occurred within 21 days after a URTI. 
TABLE 1 Baseline characteristics of the patients included in the study

Data are presented as mean \pm SD or $n(\%)$, unless otherwise stated. BMI: body mass index; COPD: chronic obstructive pulmonary disease; LABA: long-acting $\beta_{2}$-agonist; LAMA: long-acting muscarinic antagonists; mMRC: modified Medical Research Council; SGRQ: St George's Respiratory Questionnaire; CAT: COPD Assessment Test; FEV1: forced expiratory volume in $1 \mathrm{~s}$; FVC: forced vital capacity; TLC: total lung capacity; RV: residual volume; BODE: body mass index, airflow obstruction, dyspnoea, and exercise. " : score ranges from 0 to 4 , with a score of 4 indicating that the patient is too breathless to leave the house or becomes breathless when dressing or undressing; ": score ranges from 0 to 100 , with higher scores indicating worse health status and a minimum clinically important difference of 4 points; ${ }^{+}$: score ranges from 0 to 40 , with higher scores denoting a more severe impact of COPD on a patient's life and a difference between stable and exacerbation patients of 5 units. No target score represents the best achievable outcome; $\S$ : defined as a change in $\mathrm{FEV} 1 \geqslant 12 \%$ and $\geqslant 200 \mathrm{~mL}$ when comparing pre- and post-bronchodilator values.

\section{Viral infections at different visits}

The incidence of viral infections at different visits is shown in figure 2. The prevalence of individual viruses at each visit is shown in figure 3 and supplementary table S1.

Baseline visit: From the 445 patients included in the analysis, PCR was performed for 441 patients at the baseline visit, revealing a prevalence of $5.0 \%(n=22)$ of at least one respiratory virus at the time of inclusion. The viruses detected at baseline were rhinovirus $(50.0 \%)$, coronavirus $(18.2 \%)$, influenza $(9.1 \%)$, parainfluenza (4.5\%), metapneumovirus (4.5\%), RSV (4.5\%) and adenovirus (4.5\%), and one patient (4.5\%) had both coronavirus and influenza.

Stable visits: From a total of 2043 stable visits, PCR was performed at 1909 visits and revealed evidence of at least one respiratory virus in $5.3 \%(\mathrm{n}=102)$ of visits. Rhinovirus accounted for $54.2 \%$ of all viral infections and coronavirus for $20.5 \%$, followed by adenovirus (8.4\%), parainfluenza (6.5\%), influenza (5.5\%), RSV (2.8\%), bocavirus (0.9\%) and metapneumovirus (0.9\%) (supplementary table S2). None of the above viruses were identified in two consecutive stable visits (supplementary figure S1A), indicating infection rather than colonisation. However, in one case, infection with rhinovirus was followed by corona virus in two consecutive stable visits.

URTI visits: From a total of 403 URTI visits, PCR was performed in 391 visits and revealed evidence of at least one respiratory virus in $52.4 \%$ of visits. The incidence of viral infections at URTI visits was significantly higher than at stable visits $(p<0.001)$. Rhinovirus and coronavirus were the most frequently detected viruses at URTI visits, accounting for $47.7 \%$ and $24.3 \%$ of all viral infections, respectively. Influenza accounted for 9.3\%, parainfluenza for $8.7 \%$ and RSV for $7.8 \%$ of all viral infections (supplementary table S2). 


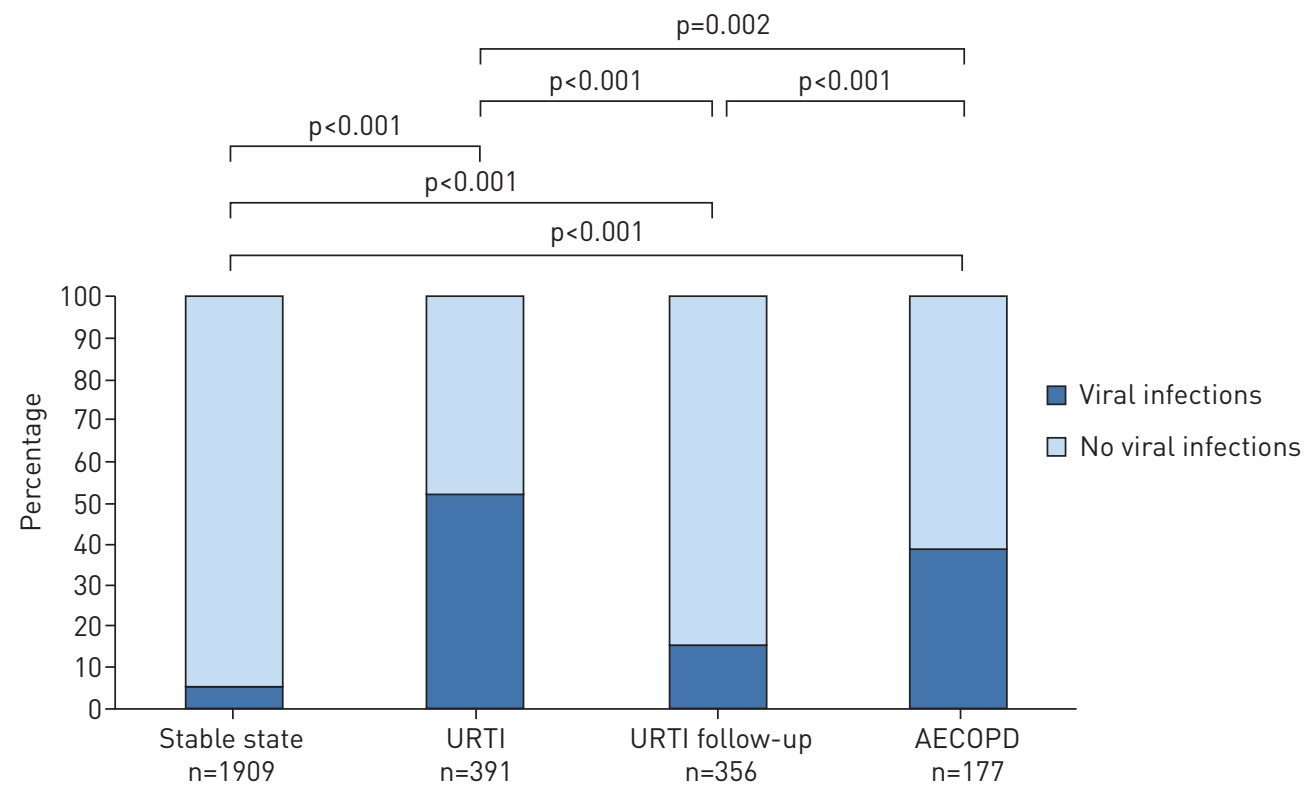

FIGURE 2 Percentages of patients with and without viral infections as assessed by PCR at different visits. URTI: upper respiratory tract infection; URTI follow-up: 10 days after URTI; AECOPD: acute exacerbation of COPD.

In one case, parainfluenza virus, and in two cases, rhinovirus, was identified both at stable state visits and at consecutive URTI visits (supplementary figure S1B). In five cases, patients with a viral infection at a stable visit also had a viral infection at a consecutive URTI visit, although not with the same virus. In one case, RSV was detected at the URTI visit and influenza in a subsequent stable visit (supplementary figure $\mathrm{S} 1 \mathrm{C})$.

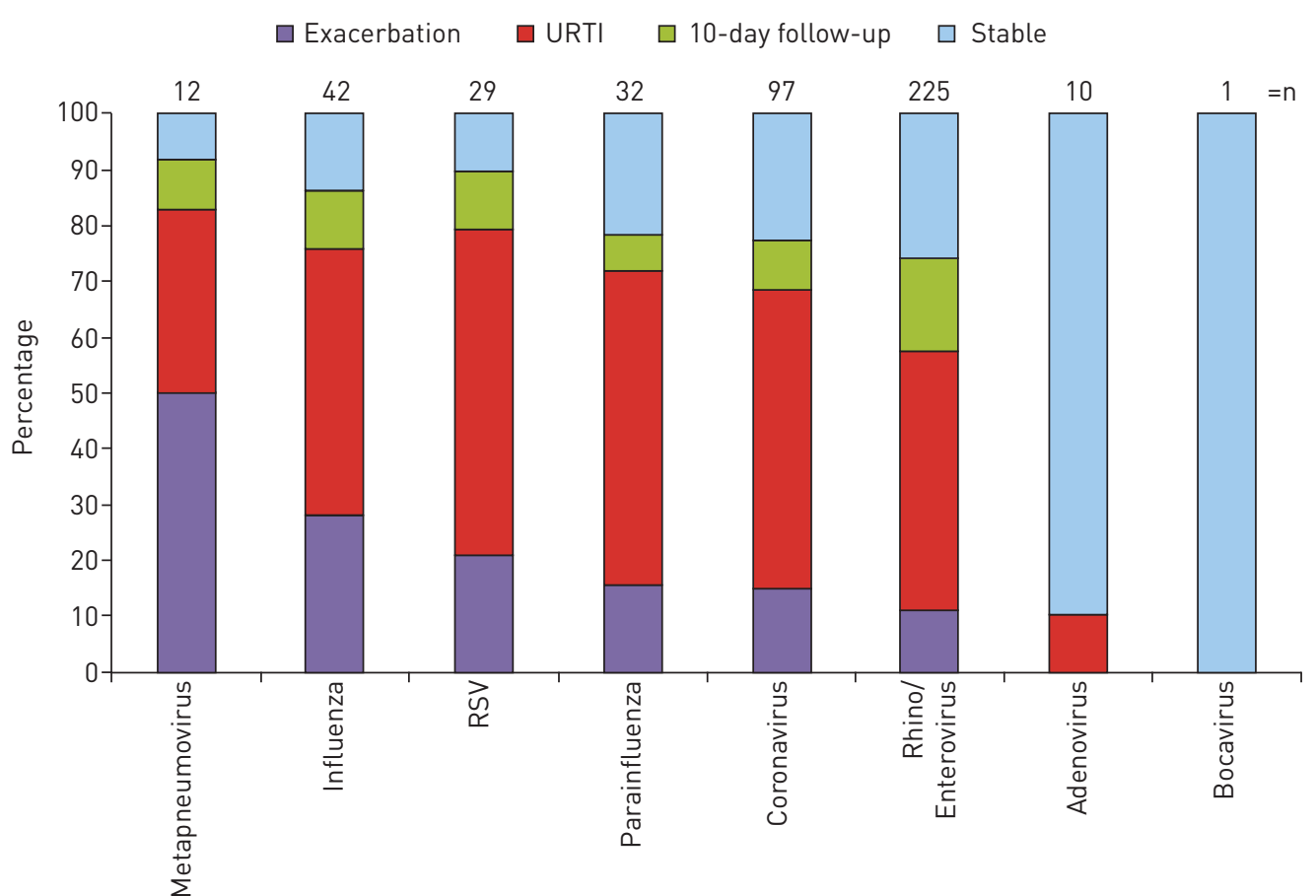

FIGURE 3 Numbers and percentages of the individual respiratory viral infections at each visit throughout the study duration. The bar charts depict the number of viruses aggregated as follows: influenza (influenza $A$ H1N1, influenza $B$, influenza $H 1$ and influenza $H 3$ ); parainfluenza (parainfluenza 1, parainfluenza 2 , parainfluenza 3, parainfluenza 4); coronavirus (coronavirus 229E, coronavirus HKU1, coronavirus NL63, coronavirus OC43); and respiratory syncytial virus (RSV A and RSV B). URTI: upper respiratory tract infection. 


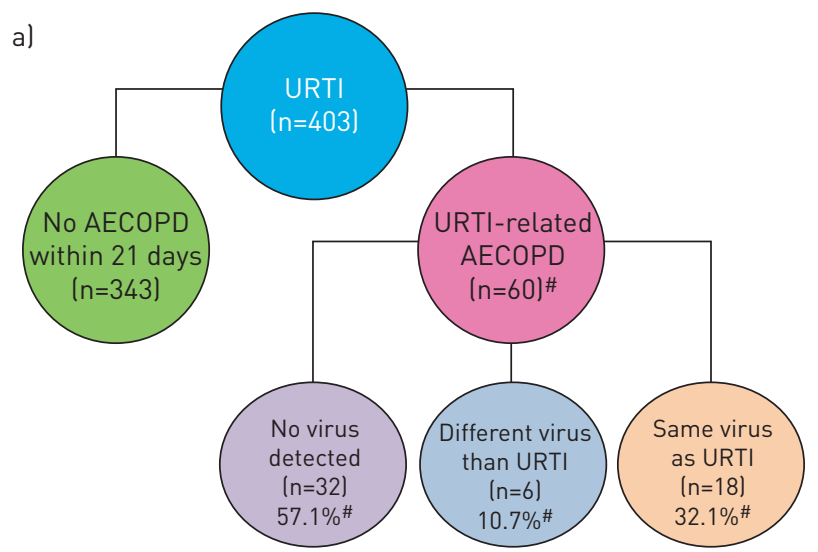

b)

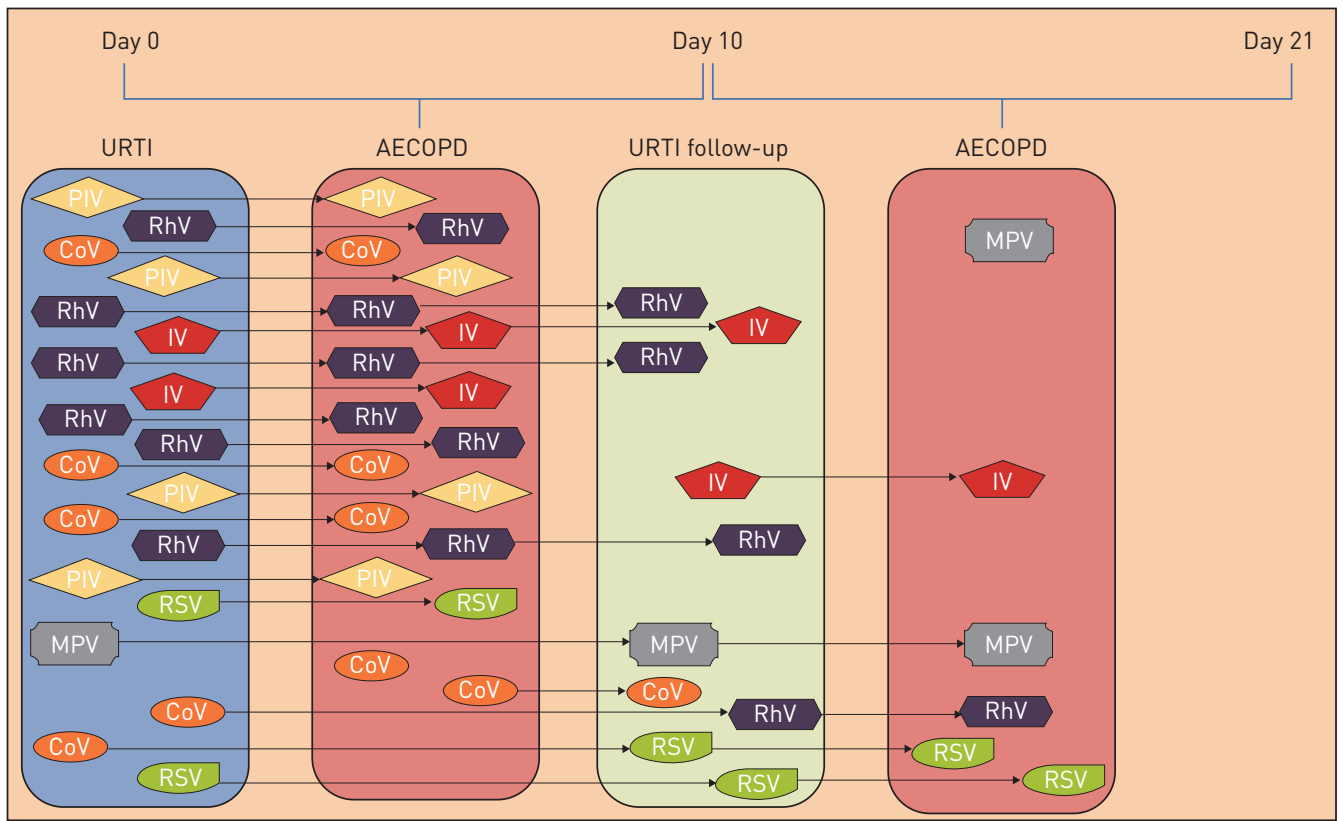

FIGURE 4 a) Number of upper respiratory tract infection (URTI)-related acute exacerbations of chronic obstructive pulmonary disease (AECOPD) loccurring within 21 days following a URTII. b) Viral infections in URTI-related AECOPD. PIV: parainfluenza virus; RhV: rhinovirus; CoV: corona virus; IV: influenza virus; RSV: respiratory syncytial virus; MPV: metapneumovirus. ${ }^{\#}$ : PCR was performed in 56 visits.

URTI-follow-up visits: From a total of 400 URTI-follow-up visits, which took place 10 days after the onset of URTI symptoms, PCR was performed in 356 visits and revealed evidence of at least one respiratory virus in $15.2 \%$ of the visits. The incidence of viral infections at URTI-follow-up visits was higher than for stable visits but lower than for URTI visits $(\mathrm{p}<0.001$ for all). Rhinovirus accounted for $67.9 \%$ of all viruses detected at URTI follow-up, followed by coronavirus (14.3\%), influenza (7.2\%), RSV (5.4\%), parainfluenza (3.6\%) and metapneumovirus (1.8\%) (supplementary table S2).

In six cases, patients with a viral infection at the URTI visit had the same viral infection at a consecutive URTI-follow-up visit (two cases with corona virus and rhinovirus, one case with influenza virus, one case with parainfluenza virus, one case with RSV and one case with rhinovirus). In one case, the patient was infected with metapneumovirus at the URTI visit and with rhinovirus at a consecutive URTI-follow-up visit (supplementary figure S1D).

AECOPD visits: From a total of 187 AECOPD visits, PCR was performed in 177 visits and revealed evidence of at least one respiratory virus in $38.4 \%$ of the visits. The incidence of viral infections at AECOPD visits was higher than for stable visits $(\mathrm{p}<0.001)$ and lower than for URTI visits $(\mathrm{p}=0.002)$. Rhinovirus and coronavirus were the most frequently detected viruses at AECOPD visits, accounting for $35.7 \%$ and $25.9 \%$ of all viral infections, respectively. Influenza accounted for $21.4 \%$, parainfluenza for 
8.6\%, RSV for $8.6 \%$ and metapneumovirus for $8.6 \%$ of all viral infections (supplementary table S2). Even though rhinovirus was the most common virus detected at all study visits, only $11.1 \%$ of all rhinovirus detections were associated with an exacerbation.

In six cases, the same virus was detected in a URTI and in a subsequent AECOPD visit; two cases with rhinovirus, one case with influenza virus, one case with parainfluenza virus, one case with RSV and one case with corona virus (supplementary figure S1E).

60 AECOPD occurred within 21 days after a URTI (figure 4a). PCR was performed in 56 of these events: in 32 of AECOPD there was no virus detected, in six there was a different virus compared with the preceding URTI and in 18 the same virus was detected as in the preceding URTI. Figure $4 \mathrm{~b}$ depicts the sequence of viral infections within 21 days following a URTI. Most URTI-related AECOPD occurred within the first 10 days after a URTI.

Patients presenting a positive viral PCR at URTI onset did not have a higher incidence of exacerbation as compared to those showing negative viral PCR results $(\mathrm{p}=0.993)$. Likewise, the incidence of any viral infection during an AECOPD was similar between AECOPD following a URTI and AECOPD independent of a URTI $(\mathrm{p}=0.206)$ (table 2). However, detection of parainfluenza 3 at URTI onset was associated with a higher risk of an AECOPD $(p=0.003)$ (table 3). There were nine positive tests for parainfluenza 3 at URTI visits, resulting in an incidence of $2.53 \%$ of parainfluenza 3 in URTI visits and accounting for $4.13 \%$ of all detected viruses in URTI visits. Of these nine parainfluenza 3-positive URTI visits, four (44.4\%) turned into an AECOPD within 21 days from the URTI.

Patients provided sputum in 124 out of 187 AECOPD cases. Of these, 108 sputum samples were of good quality and were assayed for bacterial growth. 61 samples (56.5\%) were positive for bacteria growth (few, moderate or lots of growth) and 47 samples (43.5\%) were considered negative. The bacteria that were identified were Streptococcus pneumonia in two cases (1.85\%), S. pneumonia plus Acinetobacter baumanii in one case (0.92\%), S. pneumonia plus Enterobacteriacea spp. in one case (0.92\%), Haemophilus influenzae in three cases $(2.77 \%), H$. influenza plus Moraxella catarrhalis in two cases (1.85\%), M. catarrhalis in eight cases $(7.41 \%)$, M. catarrhalis plus other in three cases $(2.77 \%)$, M. catarrhalis plus Enterobacteriace spp. plus other in one case (0.92\%), Staphylococcus aureus plus Escherichia coli in one case (0.92\%), Staphylococcus aureus in one case (0.92\%), Klebsiella pneumoniae in two cases (1.85\%), E. coli in one case (0.92\%), Enterobacteriacea spp. in 15 cases (13.89\%), Pseudomonas aeruginosa in four cases (3.7\%), $M$. catarrhalis plus Enterobacteriacea spp. in one case $(0.92 \%)$, Enterobacteriacea spp. plus other in three cases (2.77\%), P. aeruginosa plus other in one case (0.92\%), P. aeruginosa plus Achromobacter xylosoxidans in one case (0.92\%), P. aeruginosa plus Acinetobacter junii plus Enterobacteriacea spp. in one case (0.92\%), A. baumannii in three cases (2.77\%), Stenotrophomonas maltophilia in one case $(0.92 \%)$ and other in five cases (4.63).

Out of 187 AECOPD, 102 cases (54.4\%) were tested for both viruses and bacteria. Viral and bacterial infections were identified in 14 cases (13.7\%), only viral infections in 33 cases $(32.3 \%)$, only bacterial infections in 22 cases (21.6\%) and no infection in 33 cases $(32.3 \%)$.

Out of 14 cases in which both viruses and bacteria were positively identified, coronavirus infections were associated with $M$. catarrhalis (three cases, $21.6 \%$ ); influenza infections were associated with $H$. influenza (one case, 7.1\%) and with M. catarrhalis (one case, 7.1\%); rhino/enterovirus infections were associated with $H$. influenza plus $M$. catarrhalis (one case, 7.1\%), K. pneumoniae (one case, $7.1 \%$ ), S. pneumoniae (one case, $7.1 \%$ ) and $P$. aeruginosa (one case, 7.1\%); and parainfluenza infection was associated with P. aeruginosa infection (one case, $7.1 \%$ ).

\section{Factors associated with viral infections at different visits}

We further investigated how patients' characteristics as well as lung function parameters assessed by lung function tests, symptoms and quality of life questionnaires (CAT, WURSS, SGRQ) were associated with viral infections at different visits.

Stable visits: At baseline, patients with viral infections were younger and had a higher body mass index (BMI) than patients without viral infections (mean age 63 versus 67 years, $\mathrm{p}=0.048$; mean BMI 30.2 versus $27.0 \mathrm{~kg} \cdot \mathrm{m}^{-2}, \mathrm{p}=0.044$ ) (table 4, figure 5).

Insulin therapy was associated with a significantly higher incidence of viral infections. Pre- and post-bronchodilator body plethysmography data showed that patients with viral infections had significantly higher values of $\mathrm{FEV}_{1}, \mathrm{FEV}_{1} \%$ pred, FEV1/maximum vital capacity, single-breath diffusing capacity of the lung for carbon monoxide (DLCO) \% pred, and lower residual volume/total lung capacity and BODE score (BMI-airflow obstruction-dyspnoea, and exercise) (table 4, figure 5). Interestingly, patients with viral infections exhibited a higher post-bronchodilator reversibility. 
TABLE 2 Viral infections during AECOPD stratified by the presence of a URTI in the previous 21 days before the AECOPD

\begin{tabular}{lccc} 
& AECOPD following URTI & AECOPD independent of URTI & p-value \\
\hline Events $n$ & 56 & 121 & \\
Any viral infection & $24(44.6)$ & $44(36.4)$ & 0.206 \\
Adenovirus & $0(0.0)$ & $0(0.0)$ & \\
Bocavirus & $0(0.0)$ & $0(0.0)$ & 0.811 \\
Coronavirus & $5(8.9)$ & $10(8.3)$ & 0.631 \\
Influenza & $3(5.4)$ & $9(7.4)$ & 0.928 \\
Metapneumovirus & $2(3.6)$ & $4(3.3)$ & 0.054 \\
Parainfluenza & $4(7.1)$ & $1(0.8)$ & 0.341 \\
RSV & $3(5.4)$ & $3(2.5)$ & 0.851 \\
Rhino/enterovirus & $7(12.5)$ & $18(14.9)$ & \\
\hline
\end{tabular}

Data are presented as $\mathrm{n}(\%)$. Mixed logistic regression models were used to consider the effect of a patient having more than one visit. AECOPD: acute exacerbation of chronic obstructive pulmonary disease; URTI: upper respiratory tract infection; RSV: respiratory syncytial virus.

Data from all stable visits with a PCR analysis $(n=1909)$ demonstrated that cough was more commonly reported in patients with viral infections $(\mathrm{p}=0.018)$; this was confirmed by responses to the specific question in the CAT $(\mathrm{p}=0.002)$ (supplementary table S3, figure 5). Patients with viral infections also produced more sputum $(\mathrm{p}=0.041)$. In addition, there was more evidence of potentially pathogenic bacteria in sputum from patients with viral infections $(\mathrm{p}=0.026)$, particularly for $P$. aeruginosa $(\mathrm{p}=0.013)$.

In a mixed linear logistic regression model adjusted for age and sex, an increase of 10 units in SGRQ impact score at stable state was associated with an $11.6 \%$ increased chance of having any viral infection (OR 1.12, 95\% CI 1.01-1.23, $\mathrm{p}=0.032$ ).

URTI visits: During the study, there was a direct temporal association between the incidence of URTIs and AECOPD (both following a URTI and independent of the URTI) in winter months (figure 6).

\begin{tabular}{|c|c|c|c|c|c|c|}
\hline Risk factor ${ }^{\#, \eta}$ & Value & Estimate & Standard error & Standard error ratio & $\operatorname{HR}(95 \% \mathrm{CI})$ & p-value \\
\hline \multirow[t]{2}{*}{ Coronavirus 229E } & No viral infection & 0.135 & 0.290 & 1.084 & $1.145(0.65-2.02)$ & 0.641 \\
\hline & Coronavirus 229E & -0.529 & 1.033 & 1.015 & $0.589(0.08-4.46)$ & 0.608 \\
\hline \multirow[t]{2}{*}{ Coronavirus HKU1 } & No viral infection & 0.169 & 0.290 & 1.074 & $1.184(0.67-2.09)$ & 0.561 \\
\hline & Coronavirus HKU1 & 0.165 & 0.787 & 1.068 & $1.179(0.25-5.51)$ & 0.834 \\
\hline \multirow{2}{*}{ Coronavirus $0 \mathrm{C} 43$} & No viral infection & 0.182 & 0.296 & 1.085 & $1.199(0.67-2.14)$ & 0.539 \\
\hline & Coronavirus $0 \mathrm{C} 43$ & 0.257 & 0.640 & 1.046 & 1.293 (0.37-4.53) & 0.688 \\
\hline \multirow[t]{2}{*}{ Influenza B } & No viral infection & 0.202 & 0.286 & 1.058 & $1.224(0.70-2.14)$ & 0.480 \\
\hline & Influenza B & 1.006 & 0.839 & 1.128 & $2.734(0.53-14.10)$ & 0.231 \\
\hline \multirow[t]{2}{*}{ Metapneumovirus } & No viral infection & 0.181 & 0.290 & 1.083 & $1.198(0.68-2.12)$ & 0.533 \\
\hline & Metapneumovirus & 1.039 & 0.889 & 0.865 & $2.827(0.49-16.10)$ & 0.242 \\
\hline \multirow[t]{2}{*}{ RSV A } & No viral infection & 0.164 & 0.289 & 1.081 & $1.178(0.67-2.08)$ & 0.571 \\
\hline & RSV A & 0.172 & 1.020 & 0.994 & $1.187(0.16-8.76)$ & 0.866 \\
\hline \multirow[t]{2}{*}{ RSV B } & No viral infection & 0.190 & 0.282 & 1.046 & $1.210(0.70-2.10)$ & 0.500 \\
\hline & RSV B & 0.625 & 0.794 & 1.081 & $1.869(0.39-8.86)$ & 0.431 \\
\hline \multirow[t]{2}{*}{ Rhino/enterovirus } & No viral infection & -0.129 & 0.336 & 1.122 & $0.879(0.45-1.70)$ & 0.701 \\
\hline & Rhino/enterovirus & -0.647 & 0.437 & 1.153 & $0.524(0.22-1.23)$ & 0.139 \\
\hline
\end{tabular}

URTI: upper respiratory tract infection; HR: hazard ratio; RSV: respiratory syncytial virus. * : regressions for which the risk factor was adenovirus, bocavirus, influenza A H1N1, influenza H1 subtype, influenza H3 subtype, parainfluenza 2 and parainfluenza 3 did not converge due

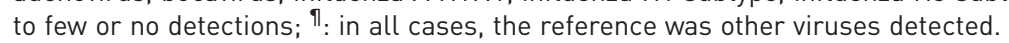


TABLE 4 Descriptive statistics for characteristics at baseline visits

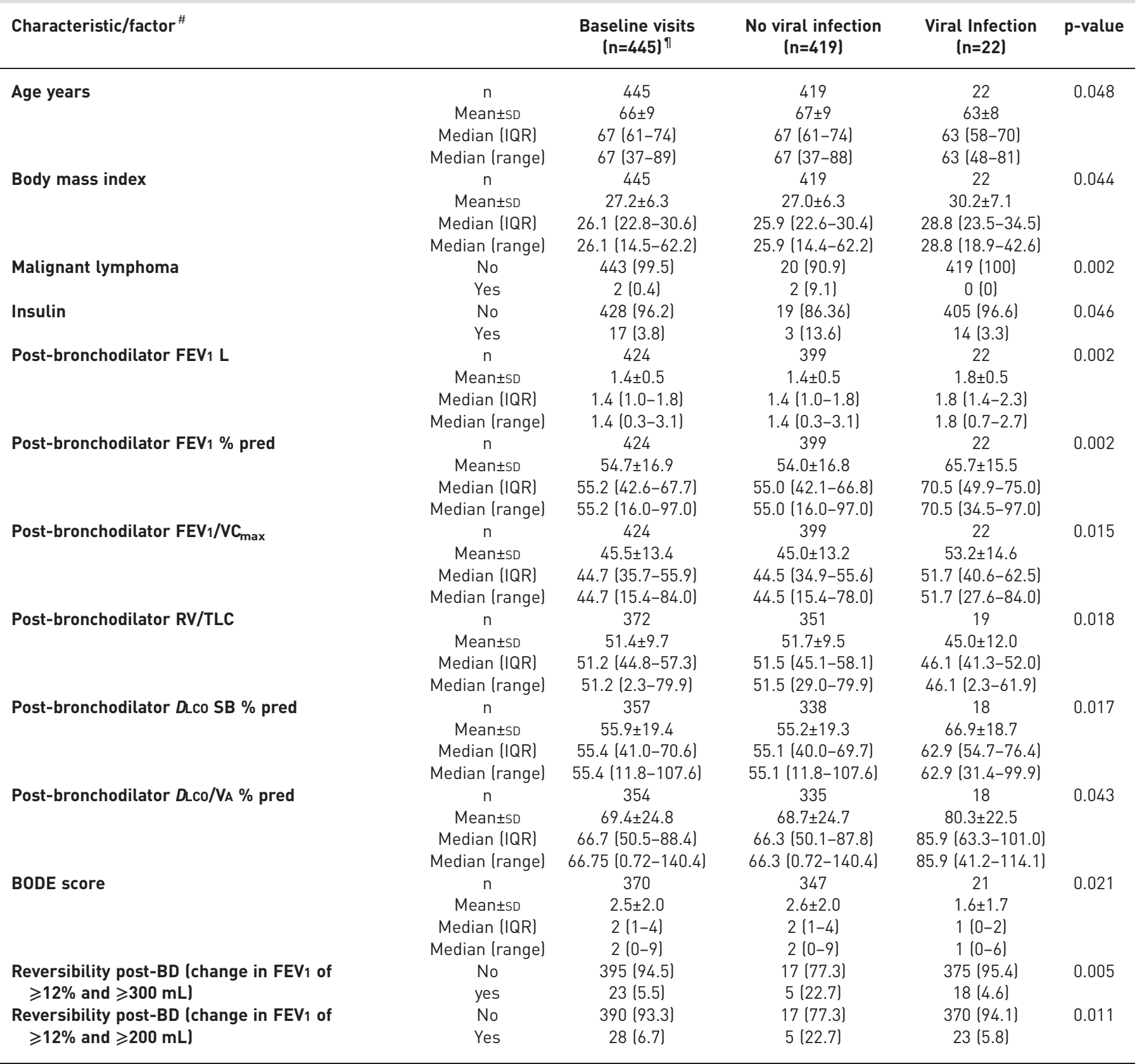

Lung function parameters were also assessed pre-bronchodilation and values were similar to those presented for post-bronchodilation.

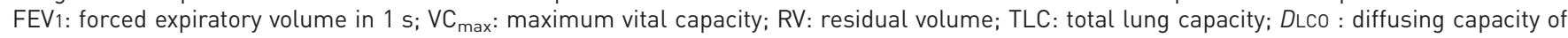
the lung for carbon monoxide; SB: single breath; VA: alveolar volume; BODE: body mass index, airflow obstruction, dyspnoea, and exercise; BD: bronchodilator; IQR: interquartile range. " : among the parameters that were tested (sex, smoking status, shortness of breath, cough, wheezing, sputum production, inhaled medication, systemic medication, oxygen therapy, noninvasive ventilation, surgical therapy for COPD, comorbidities, modified Medical Research Council dyspnoea scale, St George's Respiratory Questionnaire, COPD Assessment Test score, microbiology in sputum, Global Initiative for Obstructive Lung Disease (GOLD) stage and group, and lung function parameters including SB, NO in exhaled air and 6-min walking distance), only statistically significant parameters are presented. ": four patients had no information on viral infection.

The median annual rate (minimum to maximum) per number of patients was 0.29 (0.13-0.43) for URTIs and $0.12(0.09-0.18)$ for AECOPD.

In a mixed linear logistic regression model adjusted for age and sex, an increase of 10 units in WURSS-21 score at URTI onset was associated with a 15\% increased chance of having any viral infection (OR 1.15, 


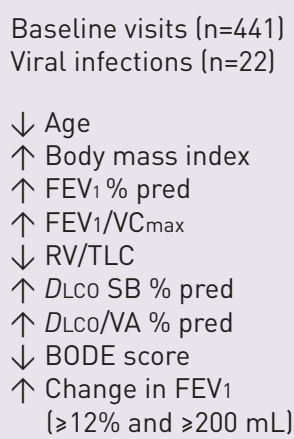

FIGURE 5 Factors significantly associated with viral infections at different visits. FEV1: forced expiratory volume in $1 \mathrm{~s} ; \mathrm{VC}_{\text {max }}$ : maximum vital capacity; $\mathrm{RV}$ : residual volume; TLC: total lung capacity; DLCo: diffusing capacity of the lung for carbon monoxide; SB: single breath; $V_{A}$ : alveolar volume; BODE: body mass index, airflow obstruction, dyspnoea, and exercise; CAT: COPD Assessment Test. \#: including baseline visits.

95\% CI 1.04-1.27, $\mathrm{p}=0.003$ ), a $24.6 \%$ increased chance of having an influenza infection (OR 1.246, 95\% CI $1.004-1.546, \mathrm{p}=0.041)$ and a $35.0 \%$ increased chance of having a parainfluenza infection (OR 1.35, 95\% CI 1.07-1.71, $\mathrm{p}=0.012$ ) (supplementary table $\mathrm{S} 4$, results shown are for a one-unit increase).

At URTI follow-up, viral infections were significantly associated with the age of patients after adjustment for sex and SGRQ symptoms score, SGRQ activity score, SGRQ impacts score, SGRQ total score, CAT, mMRC dyspnoea scale, WURSS-21 score, FEV1 \% pred and FeNO measurement ( $<<0.005$ for all). After adjustment for age and sex, an increase of 10 units in WURSS-21 score at URTI follow-up was associated with a $23.4 \%$ increased chance of having any viral infection (OR $1.23,95 \%$ CI $1.10-1.39, \mathrm{p}=0.001$ ) and a $22.1 \%$ increased chance of having rhino/enterovirus infection (OR 1.22, 95\% CI 1.06-1.40, p=0.004) (supplementary table S5, results shown are for a one-unit increase).

We further analysed changes in symptoms between the last stable visit before the URTI and the URTI-follow-up visit, stratified by virus infection at URTI follow-up. Patients with a positive viral PCR at URTI follow-up had worse symptoms than patients with a negative viral PCR, as assessed by higher values in the mMRC $(p=0.022)$, SGRQ activity score $(p=0.028)$ and SGRQ total score $(p=0.044)$ (supplementary table S6).

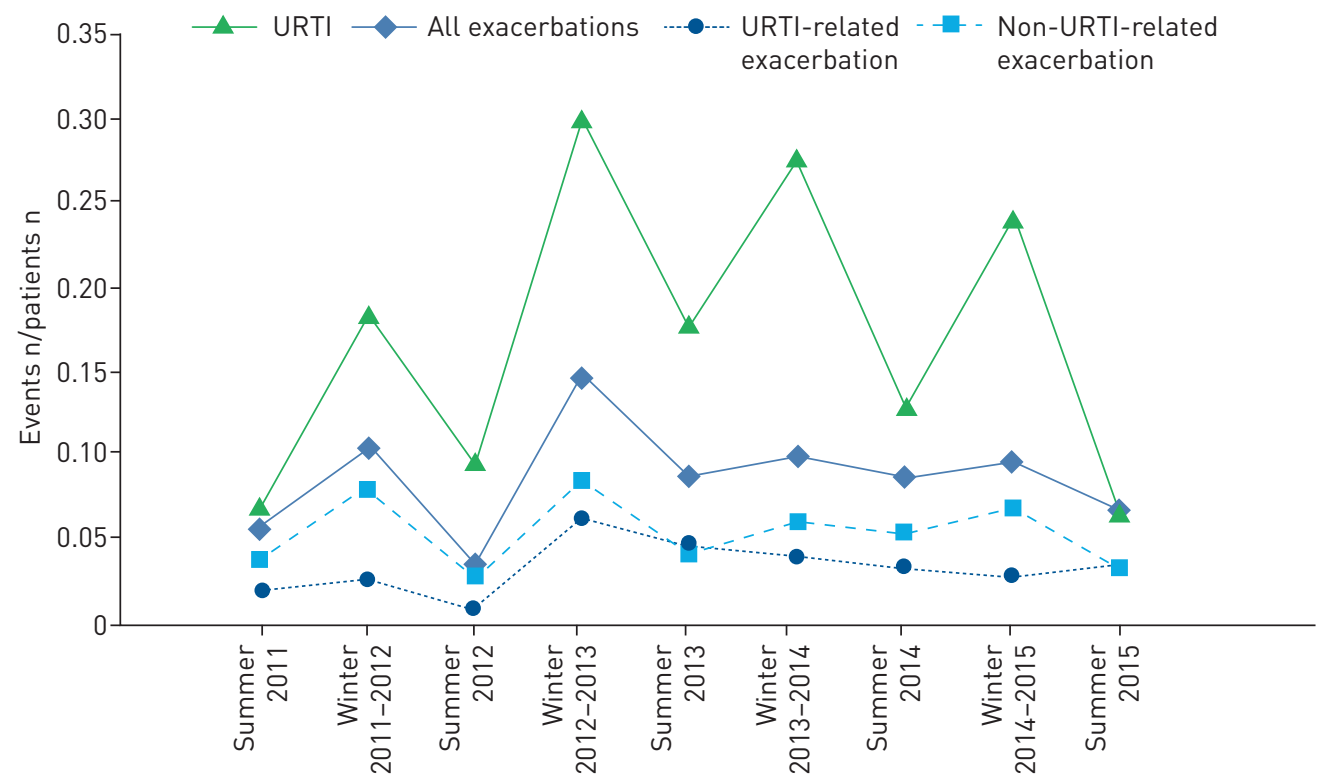

FIGURE 6 Upper respiratory tract infections (URTIs) and exacerbations over the duration of the study Incidence of URTI and chronic obstructive pulmonary disease exacerbations (following a URTI or independent of a URTI) in the overall population throughout the study according to the yearly season (summer and winter). 
AECOPD visits: Cough and sputum production were more common symptoms in patients with a viral infection during an AECOPD ( $\mathrm{p}<0.001$ and $\mathrm{p}=0.002$, respectively) (supplementary table S7, figure 5). Oedema of the extremities was more frequently present in patients without viral infections $(\mathrm{p}=0.044)$. Furthermore, patients with a viral infection during an AECOPD had a higher CAT score $(\mathrm{p}=0.007)$ and lower BODE score $(\mathrm{p}=0.008)$ than patients without viral infection (supplementary table $\mathrm{S} 7$, figure 5).

In a mixed logistic regression model adjusted for age and sex, an increase of one unit in the CAT score was associated with an $8.4 \%$ increased chance of having any viral infection at the AECOPD visit (OR 1.084, 95\% CI 1.03-1.14, $\mathrm{p}=0.005$ ). In an explorative analysis using mixed logistic regression for various associations of viral infections adjusted for the study medication, the risk of having any viral infection during an AECOPD was similar between patients that received ICS/LABA and patients that did not receive the study medication (supplementary table $\mathrm{S} 8$ ).

Evolution of AECOPD was evaluated by CAT, mMRC and WURSS-21 questionnaires at the AECOPD and follow-up visits (21 days after the AECOPD). In a linear mixed regression model adjusted for age and sex (fixed effects) and for patient (random effect), there was a decrease in CAT, mMRC and WURSS-21 scores between the AECOPD and AECOPD-follow-up visits. The decrease in CAT score between the AECOPD and AECOPD-follow-up visits was significantly higher in cases of AECOPD with viral infection compared with AECOPD cases without viral infection ( -7.12 versus $-4.28, \mathrm{p}=0.013)$.

We further explored clinically relevant changes in patients' symptoms, as assessed by changes in total SGRQ score, between the last stable visit and the next follow-up visit that took place 10 days after the URTI (figure 7). The change in SGRQ score was significantly higher when the URTI was followed by an AECOPD ( $\mathrm{p}<0.001)$. When the URTI was not followed by an AECOPD within 21 days, $46.4 \%$ of patients reported worsening of their symptoms, $14.6 \%$ of patients reported improvement of their symptoms and $29.2 \%$ of patients reported no relevant changes in their symptoms 10 days after the onset of URTI symptoms. When the URTI was followed by an AECOPD within 21 days, $60.0 \%$ of patients reported worsening of their symptoms, $5.0 \%$ of patients reported improvement of their symptoms and $18.3 \%$ of patients reported no relevant changes in their symptoms.

All patients who had a URTI showed a significant deterioration of lung function parameters irrespective of whether the URTI was followed by an AECOPD or not (figure 8).

\section{Discussion}

The present study analysed the predefined co-primary aim of the PREVENT study [13] regarding a longitudinal profiling of viral infections in COPD patients and their implications for URTI, AECOPD,

a)

\begin{tabular}{lccc}
\hline Symptoms & $\begin{array}{c}\text { URTI without subsequent } \\
\text { AECOPD within 21 days }\end{array}$ & $\begin{array}{c}\text { URTI with subsequent } \\
\text { AECOPD within 21 days }\end{array}$ & -value \\
\hline Events $\mathrm{n}$ & 343 & 60 & \\
Change in SGRQ median (minimum-maximum) & $4.3(-23.8-47.1)$ & $11.3(-14.0-66.0)$ & $<0.001^{\#}$ \\
Clinically relevant worsening in symptoms & $159(46.4)$ & $36(60.0)$ & 0.0219 \\
Clinically relevant improvement in symptoms§ & $50(14.6)$ & $3(5.0)$ & \\
No clinically relevant change in symptomsf & $100(29.2)$ & $11(18.3)$ \\
Missing cases (no SGRQ) & $34(9.9)$ & $10(16.7)$ & \\
\hline
\end{tabular}

b)

Change in SGRQ from last stable visit to URTI follow-up visit

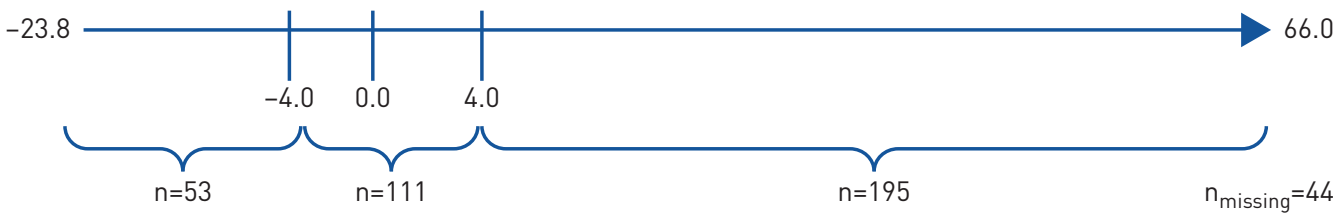

FIGURE 7 a) Clinically relevant changes in symptoms between last stable visit and upper respiratory tract infection (URTI) follow-up visit, 10 days after a URTI, as assessed by a St George's Respiratory Questionnaire (SGRQ) score change of 4 points. Data are presented as $n$ (\%), unless otherwise stated. AECOPD: acute exacerbation of chronic obstructive pulmonary disease. "\#: Mann-Whitney U-test; ": Chi-squared test; ${ }^{+}$: increase in SGRQ $\geqslant 4$ points (from 4.0 to $66.0, n=195$ ); ${ }^{\S}$ : decrease in $S G R Q \leqslant 4$ points (from -23.8 to -4.0 , $\mathrm{n}=53$ ); ${ }^{f}$ : change in SGQR between 1 and 3 (from $>-4.0$ to <4.0, $\mathrm{n}=111$ ). b) Change in SGRQ from last stable visit to URTI follow-up visit. 
symptoms and physiological changes. We found no viral colonisation in COPD patients at stable state and none of the viruses detected at URTI, except parainfluenza 3, was per se associated with an increased risk of exacerbation. Patients with a positive viral PCR at URTI onset did not have a higher incidence of exacerbation than patients with a negative viral PCR.

Invasion of respiratory viruses into the peripheral airways of COPD patients has been well documented during exacerbations as well as in stable conditions [16, 17]. It has been suggested that viral colonisation may play a role in maintaining the airway inflammation associated with stable COPD $[17,18]$. In our study, the incidence of at least one respiratory virus at stable state was low (5.3\%), with rhinovirus and corona virus being the most common viruses accounting for $54.2 \%$ and $20.5 \%$ of all viruses, respectively. The same viruses were also found in a previous study of 68 COPD patients with stable disease, although with a higher prevalence (16.2\%) [10]. Interestingly, none of the viruses that we identified in stable state were detected in two consecutive visits, indicating that there was no viral colonisation in COPD patients at stable state.

Recent data indicate that respiratory viral infections influence the bacterial microbiome in COPD patients and vice versa. Infection of COPD subjects and healthy controls with rhinovirus resulted in an increased bacterial load in sputum and a significant prevalence of $H$. influenzae [19]. The authors suggested that infection with rhinovirus alters the respiratory microbiome and may induce secondary bacterial infections. Similar findings were also reported by MALLia et al. [18], who observed that $60 \%$ of COPD patients experienced a secondary bacterial infection after being infected with rhinovirus. In line with this evidence, in the present study we found that patients with a positive viral PCR at stable state visits had a higher incidence of potential pathogenic bacteria, particularly P. aeruginosa.

Adenovirus is known to cause acute airway infections as well as latent infections in the airway epithelium. It has been shown that the expression of adenovirus proteins is increased in airway epithelial cells obtained from subjects with irreversible airflow limitation, compared to epithelial cells from subjects without airflow limitations [20]. Furthermore, studies in guinea pigs have shown that the emphysematous changes induced by cigarette smoke are exaggerated with latent adenoviral infection [21]. These studies imply that latent adenoviral infection is a possible causal factor for COPD. In our study, however, adenovirus was identified in only $0.47 \%$ of all stable visits and was never detected in two subsequent visits, indicating that there was no colonisation. Similarly, from 102 cases with a viral infection at stable state, only two cases with rhinovirus and one case with parainfluenza developed a consequent URTI with the same virus.

As expected, the prevalence $(52.4 \%)$ of viral infections at URTI visits was significantly higher than in stable state visits. Rhinovirus, the major cause of the common cold in the community, was the predominant virus detected in URTIs; however, rhinovirus was only detected in one case in a follow-up visit, 10 days after the URTI, confirming the absence of colonisation with rhinovirus in COPD patients. Similar detection rates have also been reported in previous epidemiological studies showing that $20 \%-40 \%$ of patients with classic URTI symptoms fail to yield an aetiological agent even when using the most up-to-date viral culture and PCR techniques [22]. Therefore, we postulate that URTI symptoms may be triggered by other factors, such as allergic rhinitis. It has been suggested that allergens stimulate T-helper 2 cell pathways, resulting in eosinophilia, and that such an allergic inflammation influences, in a synergistic

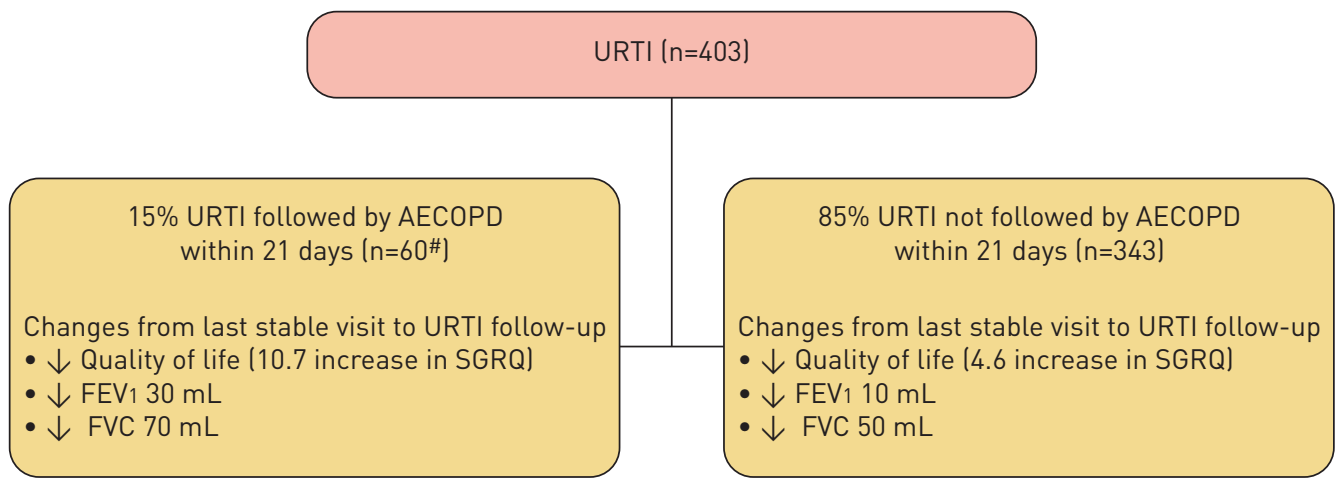

FIGURE 8 The impact of upper respiratory tract infections (URTIs) on quality of life and lung function parameters. AECOPD: acute exacerbation of chronic obstructive pulmonary disease; SGRQ: St George's Respiratory Questionnaire; FEV1: forced expiratory volume in $1 \mathrm{~s}$; FVC: forced vital capacity. Lung function measurements at URTI follow-up were performed in 323 cases $(80.1 \%)$ with a median elapsed time from URTI to URTI follow-up of 11 days (interquartile range: 10-12 days). \#: viruses not determined in four AECOPD. 
or additive manner, the infection with respiratory viruses [23]. Thus, it is tempting to hypothesise that COPD patients with eosinophilia are more susceptible to viral infections and this may explain why these patients experience more exacerbations.

The importance of colds in COPD exacerbation risk has been previously emphasised [6]; however, a longitudinal approach has been lacking. In the present study, we provide evidence that only $14 \%$ of URTIs were followed by an AECOPD within 21 days. A viral infection was detected in $43 \%$ of URTI-related AECOPD, but the same virus was detected in the AECOPD and URTI in only $32.1 \%$ of the cases. Even if the incidence of viral infections at the AECOPD visit was 38.4\%, in agreement with previous studies [6, 24], there was no difference in the incidence of any viral infection during AECOPD that followed a URTI and AECOPD independent of URTIs. It is also striking that none of the viruses detected during an URTI, except parainfluenza 3, was per se associated with an increased risk of exacerbation. Therefore, the notion that viral AECOPD are usually linked to colds could not be proven in our study. However, viral infections in URTIs may cause acute cell damage, release of reactive oxygen species and activation of NF-kB, which may lead to non-viral AECOPD [25, 26].

Rhinovirus was the most prevalent virus found during an AECOPD, accounting for $35.7 \%$ of all viral infections. This agrees with a systematic review of 19 original articles reporting on 1728 patients with AECOPD [27]. Even though rhinovirus was the most common virus detected at all study visits, only $11.1 \%$ of all rhinovirus detections were associated with an exacerbation. However, rhinovirus was the most frequently detected agent and typically circulates during the summer season, when exacerbations were less frequent. Conversely, viruses like influenza, metapneumovirus, parainfluenza and RSV circulate more frequently during the winter season and have a propensity for infecting the lower respiratory tract [22]. Considering that in $34.9 \%$ of the events no virus was identified at either the URTI or AECOPD visits, we postulate that another viral agent, which was not among the 18 routinely tested in our PCR panel, could be responsible for the infection during both the URTI and exacerbation. Of note, patients with stable COPD rarely had a positive viral PCR and the incidence of virus detection declined abruptly within 10 days of URTI onset, supporting the notion that any results provided from samples collected with a delay of even a few days may not be representative.

Viral infections have a large impact on the quality of life of COPD patients and therefore it has been suggested that quality of life questionnaires could be used to predict viral infections in COPD patients [28, 29]. To our knowledge, the present study is the first study to demonstrate that an increase of 10 units in the SGRQ impact score in COPD patients at stable state and of the WURSS-21 score during a URTI are significantly associated with an increased chance of having a viral infection. Furthermore, a one-unit increase in the CAT score was significantly associated with an increased chance of having a viral infection during an AECOPD. The CAT score has been previously shown to correlate with the length of hospitalisation in COPD patients for exacerbations, especially for patients with respiratory co-infection [24].

An SGRQ score change of 4 points between the patient's last stable visit and the next URTI-follow-up visit indicated a clinically relevant change in symptoms, as previously suggested [30]. After a URTI, about half of the patients reported a clinically relevant worsening of their symptoms, even if they did not have a subsequent AECOPD according to the current guideline definition (figure 7). These findings raise the possibility that a change in medication in COPD patients with URTI may improve outcomes.

Potential limitations of our study include the low number of exacerbations, most likely due to the fact that the diagnosis of an AECOPD was restricted to events fulfilling the criteria suggested by the official ERS/ ATS recommendations [31]. Another limitation is that we did not collect data on the vaccination status of our patients; however, it has been shown that there is no relationship between the percentage of vaccinated patients and the prevalence of influenza infections [2]. An additional limitation is that events of vasomotor rhinitis or allergic rhinitis, which present with acute symptoms similar to URTI events, may have also been included as URTI events. This could also explain the low prevalence of viral infections at URTI visits reported in our study.

Strengths of our study include the longitudinal design of the study including patients from URTI to AECOPD and the evaluation of viral detection at stable state, at URTI onset, 10 days after the URTI and during an AECOPD by multiplex PCR that detects 18 viruses, adding up to more than 50000 individual viral PCRs. Another advantage of our study was the fact that patients were instructed and equipped to collect nasal and oropharyngeal swabs at the onset of URTI symptoms, allowing for immediate sampling and thus potentially increasing PCR sensitivity. Because self-collected swabs are as sensitive as clinician-collected nasal washes for the detection of respiratory viruses by PCR [32], and because the detection of viral pathogens in oropharyngeal swabs is equally or more sensitive than in sputum samples for most viruses [33], the results presented in this study are representative for viral infections in COPD patients. Furthermore, the interventional part of the PREVENT study is not expected to influence the 
results and conclusions of the present study, given that an explorative analysis using mixed logistic regression for various associations of viral infections adjusted for the study medication showed that the risk of having any viral infection during an AECOPD was similar between patients that received ICS/ LABA and patients that did not receive the study medication.

In conclusion, our study provides solid evidence that there is a higher incidence of viral infections in URTI and AECOPD compared with the stable period of COPD. Rhinovirus was the most frequently detected virus; however, we could not confirm viral colonisation in any of the visits. The risk of exacerbation after the onset of URTI symptoms depends on the particular virus associated with the URTI, with the detection of parainfluenza 3 at URTI onset associated with a higher risk of an AECOPD. URTI is associated with clinically relevant worsening of quality of life and lung function independently of guideline-defined exacerbation. The same virus was detected in the URTI and AECOPD in only one-third of patients and, therefore, on viral detection at URTI, physicians should not assume that the same virus is likely to appear in a subsequent AECOPD. This should be considered for the effective treatment of patients as well as for the design of future studies that aim to investigate the effectiveness of antiviral treatment in COPD patients with URTIs and AECOPD. These findings have potential implications and applicability for GPs, internists and respiratory physicians when treating patients with COPD.

Author contributions: D. Stolz, H.H. Hirsch and M. Tamm conceived and designed the study. D. Stolz, D. Schilter, R. Louis, E. Papakonstantinou, M. Tamm and W. Strobel collected patient data. H.H. Hirsch organised and supervised the multiplex molecular diagnostic testing of respiratory viral pathogens. C. Schindler and L. Grize conducted the statistical analyses. All authors contributed to and approved the final manuscript, taking complete responsibility for the integrity of the work from initiation until publication.

\section{Conflict of interest: None declared.}

Support statement: The present study analyses the co-primary end point of the PREVENT study that was an investigator-initiated and driven study primarily funded by the Swiss National Foundation grant \# PP00-P3_128412/1 to D. Stolz and by the Clinic of Pneumology of the University Hospital Basel and the Department of Biomedicine, University of Basel, Switzerland. Astra AG contributed with an unrestricted grant. The corresponding author had full access to all the data in the study and had final responsibility for the decision to submit for publication. Funding information for this article has been deposited with the Crossref Funder Registry.

\section{References}

1 GBD 2017 DALYs and HALE Collaborators. Global, regional, and national disability-adjusted life-years (DALYs) for 359 diseases and injuries and healthy life expectancy (HALE) for 195 countries and territories, 1990-2017: a systematic analysis for the Global Burden of Disease Study 2017. Lancet 2018; 392: 1859-1922.

2 Seemungal T, Sykes A. Recent advances in exacerbations of COPD. Thorax 2008; 63: 850-852.

3 Kim EY, Battaile JT, Patel AC, et al. Persistent activation of an innate immune response translates respiratory viral infection into chronic lung disease. Nat Med 2008; 14: 633-640.

4 Aaron SD, Donaldson GC, Whitmore GA, et al. Time course and pattern of COPD exacerbation onset. Thorax 2012; 67: 238-243.

5 Hurst JR, Donaldson TMA, Wilkinson WR, et al. Epidemiological relationships between the common cold and exacerbation frequency in COPD. Eur Respir J 2005; 26: 846-852.

6 Johnston NW, Olsson M, Edsbäcker S, et al. Colds as predictors of the onset and severity of COPD exacerbations. Int J Chron Obstruct Pulmon Dis 2017; 12: 839-848.

7 Rohde G, Borg I, Wiethege A, et al. Inflammatory response in acute viral exacerbations of COPD. Infection 2008; 36: 427-433.

8 Hutchinson AF, Ghimire AK, Thompson MA, et al. A community-based, time-matched, case-control study of respiratory viruses and exacerbations of COPD. Respir Med 2007; 101: 2472-2481.

9 Seemungal T, Harper-Owen R, Bhowmik A, et al. Respiratory viruses, symptoms, and inflammatory markers in acute exacerbations and stable chronic obstructive pulmonary disease. Am J Respir Crit Care Med 2001; 164: 1618-1623.

10 Sethi S, Murphy TF. Infection in the pathogenesis and course of chronic obstructive pulmonary disease. $N$ Engl J Med 2008; 359: 2355-2365.

11 Falsey AR, Hennessey PA, Formica MA, et al. Respiratory syncytial virus infection in elderly and high-risk adults. N Engl J Med 2005; 352: 1749-1759.

12 Hamelin ME, Cote S, Laforge J, et al. Human metapneumovirus infection in adults with community-acquired pneumonia and exacerbation of chronic obstructive pulmonary disease. Clin Infect Dis 2005; 41: 498-502.

13 Stolz D, Hirsch HH, Schilter D, et al. Intensified therapy with inhaled corticosteroids and long-acting $\beta_{2}$-agonists at the onset of upper respiratory tract infection to prevent chronic obstructive pulmonary disease exacerbations. A multicenter, randomized, double-blind, placebo-controlled trial. Am J Respir Crit Care Med 2018; 197: 1136-1146.

14 Beckmann C, Hirsch HH. Comparing Luminex NxTAG-Respiratory Pathogen Panel and RespiFinder-22 for multiplex detection of respiratory pathogens. J Med Virol 2016; 88: 1319-1324.

15 Podbielski A, Herrmann M, Kniehl E, et al. Mikrobiologisch-infektiologische Qualitätsstandard (MiQ) 2. Auflage 2010. Munich, Urban and Fischer Elsevier GmbH, 2010.

16 Wedzicha JA. Role of viruses in exacerbations of chronic obstructive pulmonary disease. Proc Am Thor Soc 2004; 1: 115-120. 
17 Utokaparch S, Sze MA, Gosselink JV, et al. Respiratory viral detection and small airway inflammation in lung tissue of patients with stable, mild COPD. COPD 2012; 2: 197-203.

18 Mallia P, Footitt J, Sotero R, et al. Rhinovirus infection induces degradation of antimicrobial peptides and secondary bacterial infection in chronic obstructive pulmonary disease. Am J Respir Crit Care Med 2012; 186: $1117-1124$

19 Molyneaux P, Mallia P, Cox MJ, et al. Outgrowth of the bacterial airway microbiome after rhinovirus exacerbation of chronic obstructive pulmonary disease. Am J Respir Crit Care Med 2013; 188: 1224-1231.

20 Hogg JC. Role of latent viral infections in chronic obstructive pulmonary disease and asthma. Am J Respir Crit Care Med 2001; 164: S71-S75.

21 Higashimoto Y, Elliot WM, Behzad AR, et al. Inflammatory mediator mRNA expression by adenovirus E1A-transfected bronchial epithelial cells. Am J Respir Crit Care Med 2002; 166: 200-207.

22 Gwaltney JM. Clinical significance and pathogenesis of viral respiratory infections. Am J Med 2002; 112: Suppl. $6 \mathrm{~A}, 13 \mathrm{~S}-18 \mathrm{~S}$

23 Edwards MR, Strong K, Cameron A, et al. Viral infections in allergy and immunology: how allergic inflammation influences viral infections and illness. J Allergy Clin Immunol 2017; 140: 909-920.

24 Dai MY, Qiao JP, Xu YH, et al. Respiratory infectious phenotypes in acute exacerbation of COPD: an aid to length of stay and COPD Assessment Test. Int J Chron Obstruct Pulmon Dis 2015; 10: 2257-2263.

25 Teran LM, Johnston SL, Schroder JM, et al. Role of nasal interleukin-8 in neutrophil recruitment and activation in children with virus-induced asthma. Am J Respir Crit Care Med 1997; 155: 1362-1366.

26 Grunberg K, Smits HH, Timmers MC, et al. Experimental rhinovirus 16 infection. Effects on cell differentials and soluble markers in sputum in asthmatic subjects. Am J Respir Crit Care Med 1997; 156: 609-616.

27 Zwaans WA, Mallia P, van Winden ME, et al. The relevance of respiratory viral infections in the exacerbations of chronic obstructive pulmonary disease-a systematic review. J Clin Virol 2014; 61: 181-188.

28 Barrett B, Brown R, Voland R, et al. Relations among questionnaire and laboratory measures of rhinovirus infection. Eur Respir J 2006; 28: 358-363.

29 Barrett B, Brown RL, Mundt MP, et al. Validation of a short form Wisconsin Upper Respiratory Symptom Survey (WURSS-21). Health Qual Life Outcomes 2009; 7: 76.

30 Jones PW, Beeh KM, Chapman KR, et al. Minimal clinically important differences in pharmacological trials. Am J Respir Crit Care Med 2014; 189: 250-255.

31 Celli BR, MacNee W. Standards for the diagnosis and treatment of patients with COPD: a summary of the ATS/ ERS position paper. Eur Respir J 2004; 23: 932-46.41.

32 Fisher CE, Boeckh M, Jerome KR, et al. Evaluating addition of self-collected throat swabs to nasal swabs for respiratory virus detection. J Clin Virol 2019; 115: 43-46.

33 Huijskens EG, Rossen JW, Kluytmans JA, et al. Evaluation of yield of currently available diagnostics by sample type to optimize detection of respiratory pathogens in patients with a community-acquired pneumonia. Influenza Other Respir Viruses 2014; 8: 243-249. 